\title{
Clonorchis sinensis, an oriental liver fluke, as a human biological agent of cholangiocarcinoma: a brief review
}

\author{
Tong-Soo Kim ${ }^{1}$, Jhang Ho Pak ${ }^{2}$, Jong-Bo $\mathrm{Kim}^{3}$ \& Young Yil Bahk ${ }^{3, *}$ \\ ${ }^{1}$ Department of Parasitology and Tropical Medicine, School of Medicine, Inha University, Incheon 22212, ${ }^{2}$ Department of Convergence \\ Medicine, College of Medicine, University of Ulsan, Asan Institute for Life Sciences, Asan Medical Center, Seoul 05505, ${ }^{3}$ Department of \\ Biotechnology, Konkuk University, Chungju 27478, Korea
}

Parasitic diseases remain an unarguable public health problem worldwide. Liver fluke Clonorchis sinensis is a high risk pathogenic parasitic helminth which is endemic predominantly in Asian countries, including Korea, China, Taiwan, Vietnam, and the far eastern parts of Russia, and is still actively transmitted. According to the earlier $8^{\text {th }}$ National Survey on the Prevalence of Intestinal Parasitic Infections in 2012, C. sinensis was revealed as the parasite with highest prevalence of $\mathbf{1 . 8 6} \%$ in general population among all parasite species surveyed in Korea. This fluke is now classified under one of the definite Group 1 human biological agents (carcinogens) by International Agency of Research on Cancer (IARC) along with two other parasites, Opisthorchis viverrini and Schistosoma haematobium. C. sinensis infestation is mainly linked to liver and biliary disorders, especially cholangiocarcinoma (CCA). For the purposes of this mini-review, we will only focus on $C$. sinensis and review pathogenesis and carcinogenesis of clonorchiasis, disease condition by $C$. sinensis infestation, and association between C. sinensis infestation and CCA. In this presentation, we briefly consider the current scientific status for progression of CCA by heavy $C$. sinensis infestation from the food-borne trematode and development of CCA. [BMB Reports 2016; 49(11): 590-597]

\section{INTRODUCTION}

Cholangiocarcinoma (CCA) with features of cholangiocyte differentiation is one of the main histological types of malignant tumors of biliary tract epithelia and is a relatively rare type of liver cancer (1). The only therapy for CCA is

*Corresponding author. Tel: +82-43-840-3903; Fax: +82-43-8523616; E-mail: bahk12@empal.com, byoung1@kku.ac.kr

https://doi.org/10.5483/BMBRep.2016.49.11.109

Received 4 July 2016

Keywords: Cholangiocarcinoma, Clonorchiasis, Clonorchis sinensis, Liver cancer, Liver flukes surgical operation or liver transplantation. Usually, CCA is diagnosed at advanced stages and is considered as an incurable and lethal cancer with poor survival rate of $<24$ months (2). This intimidatory cancer develops in the epithelial cells which line the bile ducts and occur in the bile ducts within the liver (intrahepatic), the bile ducts just outside the liver (perihilar) and distal bile ducts. However, this rare tumor is exceptional in regions within Asia, including northeastern Thailand and many areas of southeastern Asia, where infestation with two liver flukes, Opisthorchis viverrini and Clonorchis sinensis is widespread, respectively (3-5). Due to higher prevalence of liver flukes (a common parasitic infestation) in these areas, there is a higher incidence of CCA (6-8). Therefore, infestations with the two liver flukes are now classified under definite Group 1 biological agents (carcinogens) by the International Agency of Research on Cancer (IARC) based on sufficient evidences in humans $(3,9)$. Nowadays, three helminth infestations by two food-borne liver flukes, O. viverrini and C. sinensis and Schistosoma haematobium associated with urinary bladder cancer, have been classified under definite group 1 carcinogens. Disease conditions caused by O. viverrini and C. sinensis infestations are referred to as opisthorchiasis and clonorchiasis, respectively. Although opisthorchiasis and clonorchiasis are the well-known main risk factors of CCA, chronic infection with hepatitis $\mathrm{B}$ and $\mathrm{C}$ viruses, liver cirrhosis, chronic non-alcoholic liver disease, obesity and hepatolithiasis (gallstones) are also the other minor known risk factors (10). In fact, the connection between CCA and these liver flukes has been the subject of clinical attention for more than 60 years $(11,12)$. As experimental and epidemiological evidences accumulated, C. sinensis infestation strongly implicated the detrimental etiology of CCA with pooled odds ratios between 4.5 and $6.1(6,10,13-15)$. In this mini-review, we will limit our focus on the association between $C$. sinensis infestation and CCA by briefly summarizing the recent significant scientific progresses (for comprehensive review on O. viverrini please refer to work by Sripa B et al.). 


\section{LIFE CYCLE OF C. sinensis, SYMPTOMS, DIAGNOSIS AND EPIDEMIOLOGY}

C. sinensis is a leaf-shaped slender digenetic trematode, measuring 15-20 $\mathrm{mm}$ in length and 3-4 $\mathrm{mm}$ in width belonging to class Trematoda, phylum Platyhelminthes. This oriental or Chinese liver fluke is the most pivotal species of food-borne zoonotic parasite in East Asia including Korea, China (except for northwestern regions), Taiwan, northern Vietnam and the far eastern part of Russia, where it is still actively transmitted (17). The life cycle of C. sinensis is characterized by an alternation of sexual and asexual reproductions in three different hosts, such as snails, fish and mammals $(18,19)$. Embryonated eggs laid by hermaphroditical adult worms are discharged in the biliary ducts and stool of a definite human host. The discharged eggs ingested by a suitable snail intermediate host release miracidia, which go through some developmental stages, such as sporocysts, rediae and cercariae in a regular sequence. The finally developed cercariae in the infected snail are shed into water. These larval stages in the snails reproduce asexually and this reproduction allows for an exponential multiplication of cercariae from a miracidium. After a short period of free-swimming time in water, the shed cercariae meet the $2^{\text {nd }}$ intermediate cyprinid fish, invade the mucous skin and become encysted metacercariae in the subcutaneous tissues or muscles. When a definite mammal host including humans, cats, mink, badgers, rats and dogs eats insufficient cooked, salted, pickled, dried or smoked infested fish, metacercariae separates from the flesh through gastric juice digestion and excyst in the duodenum by a combined action of trypsin and cysteine proteases. Subsequently, the excysted flukes migrate to intrahepatic bile duct through the ampulla of Vater, develop into adult flukes and can dwell for up to 30 years. One worm in human host produces approximately 4000 eggs a day by sexual reproduction (20).

Despite several pathological changes, most of the patients with clonorchiasis in a manner similar to most of the human parasitic infestations have asymptomatic or mild non-specific symptoms except for increased frequency of palpable liver, such as asthenia, nausea, indigestion, headache, dizziness, vertigo, abdominal discomfort, diarrhea, or abdominal pain. Asymptomatic or mild non-specific symptoms may be reflected in the host-parasite relationship, which evolves intimately and progresses in a less harmful way to its host. However, based on few case reports, clinical manifestations caused by clonorchiasis have been mainly related to worm burden (20). Typical physical symptoms of C. sinensis infestation are jaundice, hepatomegaly and liver tenderness (19). Heavy and chronic C. sinensis infestation results in various complications in the liver and biliary systems, primarily cholelithiasis, cholangitis and cholecystitis (21). Growth retardation has been reported in children with heavy infestation. In addition, it is now widely acknowledged that
C. sinensis infestation may be associated with CCA. Beyond pathogenesis induced by helminth, hygiene hypothesis and considerable investigations demonstrate sudden rise in epidemic in allergic diseases, such as asthma, anaphylaxis, allergic rhinitis and atopic dermatitis in developed countries; furthermore, this phenomenon has been reported to be lower in developing countries that show a high rate of helminth infestation (22-24). As shown in human and experimental animal models, helminthes are potent immune modulators and induce down T-cell responsiveness, which is partially due to modulation of dendritic cells (DCs) and macrophages $(M \phi)$, and dampens allergic $\mathrm{T}_{\mathrm{H}} 2$ immune responses through $\mathrm{CD} 4{ }^{+} \mathrm{CD} 25^{+}$Foxp $3^{+} \mathrm{T}_{\text {reg }}$ cells. The suppression of airway inflammation in murine asthma model through treatment of C. sinensis-derived total protein is characterized by induction of $\mathrm{CD} 4{ }^{+} \mathrm{CD} 25^{+}$Foxp $^{+}{ }^{+} \mathrm{T}_{\text {reg }}$ cell development and modulation of DC functions (25), and a specific C. sinensis-derived antigen demonstrates suppressive skin inflammation through effective mast cell inhibition in allergic and inflammatory diseases (26). It is noteworthy that parasitic helminthes stimulate some regulatory mechanisms associated with suppression of development of allergies in human and animal models and helminthes are candidates for broader therapeutic application through immune modulation by helminthes (27), although no universal mechanism has yet been elucidated.

The standard diagnosis of clonorchiasis is usually established by microscopic examination of the stool for eggs. The formalin-ether sedimentation technique is known to be more reliable than the direct-smear method for detecting the eggs in stool (28). Although some serological ELISA screening methods for adult $C$. sinensis antigens are currently available for detection of antibodies, they are not reliably used due to their considerable cross-reactivity and low specificity $(29,30)$. Application of recombinant proteins for excretory-secretory products (ESPs) of $C$. sinensis has enhanced the specificity for diagnosis of clonorchiasis (31). Various DNA-based techniques have been developed for the specific detection of $C$. sinensis (32). Recently, clonorchiasis was commonly diagnosed incidentally during radiological screening, especially by ultrasonography of the abdomen for other purpose, in view of the fact that symptoms of $C$. sinensis infestation are nonspecific in most of the case (33). Praziquantel is a powerful and effective Clonorchis-cidal drug of choice. Recently, tribendimidine, a derivative of amidantel and a broadspectrum anthelmintic agent, has been acknowledged as an effective and safe agent (34).

As mentioned above, liver fluke infestations occur in some Asian countries where people eat raw (salted, pickled, dried or smoked) or undercooked fish that are infested with these tiny parasite worms. In humans, these flukes dwell in the bile ducts and can cause bile duct cancer. The parasites closely related to risk of developing bile duct cancer are $C$. sinensis and O. viverrini. In case of C. sinensis, approximately 700 million people are at the risk of infestation and an estimated 
35 million are infested with C. sinensis (35). In Korea, according to the most recent $8^{\text {th }}$ National Survey on the Prevalence of Intestinal Parasitic Infections in 2012, C. sinensis was revealed as the parasite with highest prevalence of $1.86 \%$ in general population as compared to overall prevalence of $2.42 \%$ in $2004(36,37)$. In addition, the known endemic regions for C. sinensis, especially southern areas along Nakdong and Seomjin rivers, showed high incidence rates of CCA $(10,15,38)$. According to 2012 survey data, 0.93 million people were estimated to be infested with clonorchiasis on Korea. However, C. sinensis infestation causes one fourth of CCA cases in the endemic area, approximately $10 \%$ of CCA cases are estimated to be due to infestation with C. sinensis, and estimated CCA relative risk has been continuously rising particularly in areas hyper-endemic for $C$. sinensis infestation. In China, where food-borne parasitic infestations are on a rapid rise, C. sinensis infestations have been reported in 27 of 34 provinces and currently the national average prevalence has increased by $75 \%$ when compared to the results from the first national survey in 2003 (12.49 million people estimated to be infested with C. sinensis with $0.58 \%$ prevalence) (39). From a comparative point of view, an enhanced vulnerability to CCA in patients with $O$. viverrini infestation has been reported in Thailand (4).

\section{PATHOGENESIS AND CARCINOGENESIS}

C. sinensis causes mechanical injury and inflammation at the environs of biliary tree due to fluke activities, metaplasia of mucin-producing cells in the mucosa, progressive periductal fibrosis and hyperplasia of epithelial cells (40, Fig. 1). The severity of these changes exhibits a tendency to correlate with the duration of fluke infestation, the worm burden, and the

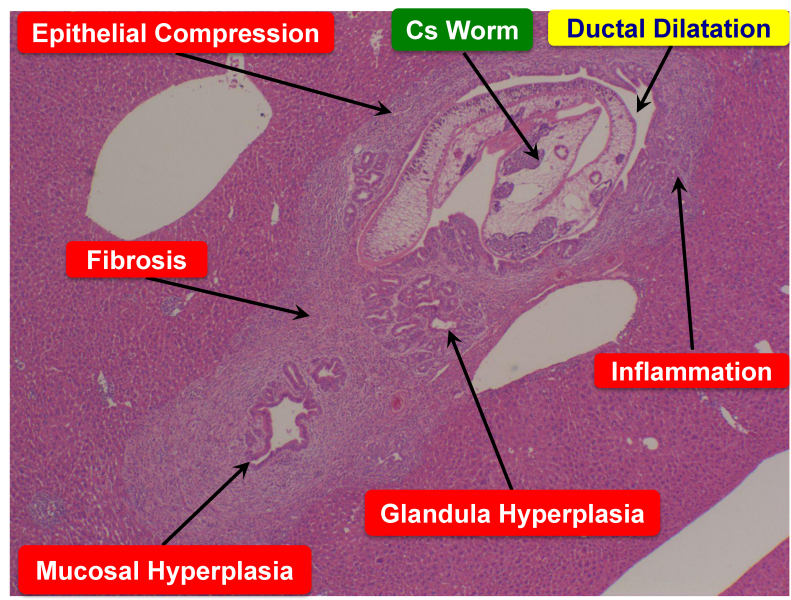

Fig. 1. Histopathological liver-section image of clonorchiasis (hematoxylin and eosin staining) at $4^{\text {th }}$ week of C. sinensis postinfestation. susceptibility of the host (41). The pathological changes and the adult flukes might be conducive as a nidus for bacterial infection and intrahepatic stone formation. In addition, the liver flukes secrete or excrete some metabolic products (so-called ESPs), which are highly immunogenic and may be toxic to or interact with the biliary epithelia to stimulate inflammation, promote proliferation and suppress apoptosis $(42,43)$. Thus, the histopathological changes originate from a combination of mechanical irritation caused by physical contact with infested worms and chemical irritation by their ESPs. Recently analyzed gene expression profiles of three developmental stages of $C$. sinensis might reflect the pathogenesis and carcinogenesis provoked by this liver fluke infestation (44).

Although the molecular mechanism involved in the development of CCA are poorly elucidated in detail, it might be simply proposed as a multistep process: normal cholangiocytes $\rightarrow$ pathogen recognition $\rightarrow$ chronic inflammation $\rightarrow$ cell damage $\rightarrow$ reactive cell proliferation $\rightarrow$ genetic/epigenetic mutations $\rightarrow$ malignant cholangiocytes in regular sequence (45). Until date, C. sinensis-induced CCA is widely acceptable to be closely linked to chronic inflammation and oxidative stress pathways for creating feasible microenvironment conducive for initiation and promotion of CCA, involving a complex process of several separate mechanisms (4, 46, 47). For pathogen recognition, Toll-like receptors (TLRs) encompass distinctive capacity to sense the initial infection and are the most potent initiators of the inflammatory responses (48). However, prolonged inflammation through excessive production of inflammatory cytokines and chemokines via TLR-mediated signaling could be detrimental because it may cause host toxicity and tissue damage. In the mouse model of clonorchiasis, the expressions of TLR2 and TLR4 were upregulated during the infestation of $C$. sinensis, indicating probable participation of TLR2 and TLR4 in the stimulation of innate immune response during $C$. sinensis infestation (49). The $T_{H} 1$-based inflammatory consequences instructed by TLRs are not only involved in eliminating pathogenic infections but can also induce fatal pathogenic consequences (50). Similarly, the $\mathrm{T}_{\mathrm{H}}$ 2-based pathogen-modulated TLR-mediated signaling event leads to development of immune response beneficial for the pathogen i.e. disease progression. During the chronic C. sinensis infestation, clonorchiasis is associated with predominant $\mathrm{T}_{\mathrm{H}} 2$ cytokine production as well as suppression of $\mathrm{T}_{\mathrm{H}} 1$ cytokine production $(51,52)$. Substantial evidences support the concept that chronic inflammation as a key feature of helminth infestation is linked to various processes involved in carcinogenesis, including cellular transformation, promotion, survival, proliferation, etc (14). In general, inflammation of the bile duct walls is only inconsiderable in regular cases. Sucking onto the biliary epithelium by the fluke results in mechanical tissue damage even at early stages of infestation and, as the fluke matures; the lesion becomes more pronounced and starts to ulcerate (53). Metaplasia of the biliary epithelial cells into 
mucin-producing cells occurs during very early C. sinensis infestation. The mucin-producing cells may proliferate to produce ESPs in the mucosa, leading to persistent and excessive mucus content in the bile (54). This event is initiated by several factors, such as mechanical obstruction of the bile ducts, mechanical injury from the physical activities of feeding and migrating worms, infestation-related inflammation including secondary infection, especially Escherichia coli, and toxic effects of ESPs $(42,43,55-59)$. Several reports demonstrated that ESPs from adult C. sinensis provoke profile changes in transcriptome, proteome and microRNA expression in human HuCCT1 CCA cells and in mouse liver $(56,58-60)$. Moreover, ESPs from C. sinensis may lead the hyperplasia of normal biliary cells to adenomatous cells with subsequent transformation into CCA by alteration of the transcriptional modification of carcinogenic target genes, such as Mcm7, through histone modifications $(16,57)$. However, the exact mechanism by which carcinogenesis occur remains to be elusive; it is hypothesized that many processes could be implicated. The following possible mechanisms of cholangiocarcinogenesis due to $C$. sinensis infestations have been postulated (16, 61): First, chronic irritation and chronic inflammation caused by the infested $C$. sinensis results in pathologic hyperplasia as a sign of abnormal or precancerous changes and adenomatous changes of bile duct epithelia. The pathologically hyperplastic cells induced by host-parasite interactions due to worm's physical activities are fragile to carcinogen because the biological agent could easily induce DNA damage during active cell proliferation. Second, endogenous oxidative and nitrative DNA damage caused by $C$. sinensis infestation has been studied in both humans and animals $(54,62,63)$. It is probable that oxidative lesion products, such as 8-nitroguanine and 8-oxo-7,8-dihydro-2'deoxyguanosine (8-OxodG), accumulate in chronic inflammation site around the bile ducts via local nitric oxide production induced by nitric oxide synthase (iNOS) (Fig. 2). Therefore, bile duct epithelial cells are exposed continuously to high concentrations of oxidative lesion contributing to CCA initiation and/or promotion $(52,64)$. Third, C. sinensis-induced redox imbalance is due to the enzymatic trigger for production of drug metabolizing enzymes and free-radical generating enzymes (65): For example, experimentally cytochrome P-450 in C. sinensis is responsible for metabolism in the worms and for detoxification contributed to worm survival and drug resistance. Also, free radicals generated by $C$. sinensis infestation play a critical role in triggering NF- $\mathrm{KB}$-mediated inflammation (57). ESPs of C. sinensis can induce recruitment of histone acetyltransferases (HAT) and regulation of minichromosome maintenance $(\mathrm{Mcm})$ proteins for the physiological hyperplasia (57). Fourth, recent evidences have shown modulation of carcinogenesis prevention processes as one of the multiple cholangiocarcinogenic pathways, for example, involvement of small non-protein-coding RNAs (microRNA). Indeed, it is now generally accepted that microRNAs serve as a negative gene regulator by participating in the modulation of a variety of physiological pathways and have the potential to control various gene targets (66). Recent finding indicates that, during C. sinensis-associated cholangiocarcinogenesis in animal model and humans, microRNAs function as both tumor suppressors and oncogenes (67). In addition, IL-6 overexpressing malignant cholangiocytes have been reported to modulate the expression of DNA methyltransferase 1 in a microRNA-dependent manner (68). In the case of carcinogenetic pathway induced by $C$. sinensis infestation, exposure of human HuCCT1 CCA cells to ESPs for different periods of time as compared to normal H69 cholangiocyte cells has shown differentially altered microRNA profile changes revealing the involvement of microRNA in cell proliferation, inflammation, oncogene activation/suppression, migration/invasion/metastasis, and DNA methylation (59).

Inflammation drives generation of free radicals (reactive oxygen species (ROS) and reactive nitrogen species (RNS)), which leads to lipid peroxidation (LPO), and promotes the acquisition of considerable oxidative DNA damage and dysregulation of cell homeostasis (Fig. 2). Considerable reports have demonstrated that ROS are involved in the link between chronic inflammation and cancer $(69,70)$. For example, exposure of human HuCCT1 CCA cells to C. sinensis ESPs revealed enhanced generation of free radicals by activation of $\mathrm{NADPH}$ oxidase (NOX), xanthine oxidase $(\mathrm{XO})$, lipoxygenase

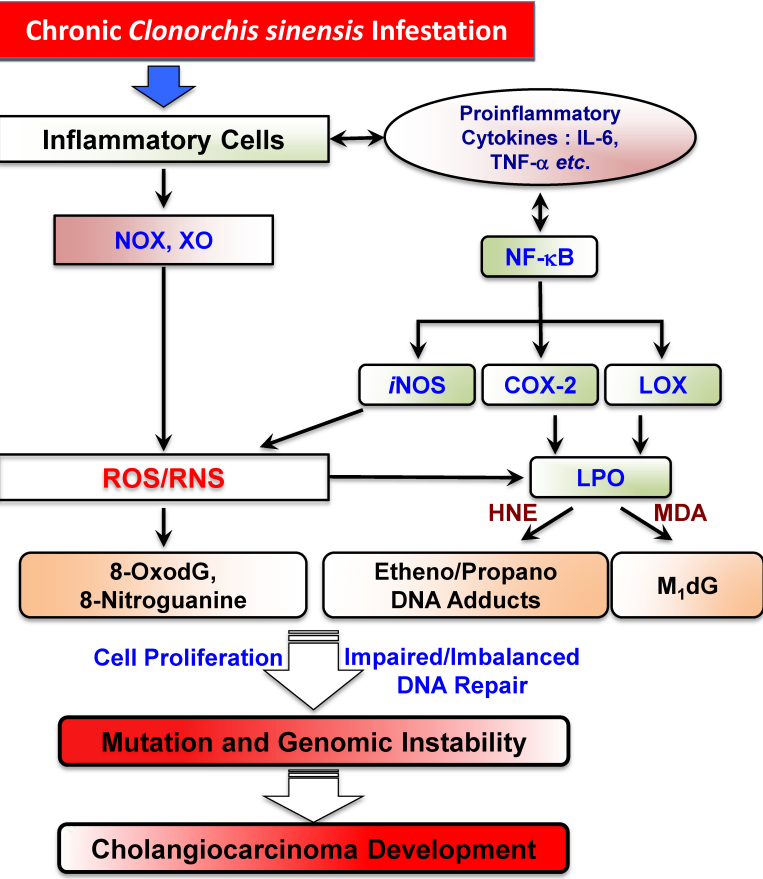

Fig. 2. Possible link of liver fluke C. sinensis-induced redox imbalance with CCA development. 
(LO), cyclooxygenase (COX) and iNOS (59). In the mouse infectious model for C. sinensis, liver fluke infestation differentially elevated secretion of proinflammatory cytokines such as TNF- $\alpha$, IL-1 $\beta$ and IL- 6 , indicating that, under the chronic inflammation states, persistent and dysregulated expressions of these pleiotropic cytokines are promutagenic for malignant cell transformation $(4,47)$. Chronic and elevated signaling events by TNF- $\alpha$ and IL- $1 \beta$ transactivation of $\mathrm{NF}-\kappa \mathrm{B}$, which in turn induces the proinflammatory mediating genes including $i N O S, I L-6$, etc, results in amplification of inflammation (71). Moreover, substantial evidences have demonstrated that nitric oxide (NO) is not only cytotoxic but may also be genotoxic leading to DNA damage. The main role of nitric oxide (synthesized by iNOS after challenge by immunological and inflammatory stimuli) during inflammation involves triggering of carcinogenesis through accumulation of DNA damage by inhibiting DNA repair system and stimulation of COX-2 expression $(72,73)$. Furthermore, LPO products, such as trans-4-hydroxy-2-nonenal (HNE), malondialdehyde (MDA) and crotonaldehyde, can modulate the $2^{\text {nd }}$ messenger systems involved in inflammation and carcinogenesis for increasing cell proliferation and decreasing apoptosis in the initiated cell population $(74,75)$. Additional critical connection between chronic inflammation and cancer development is cyclooxygenase (COX)- and lipoxygenase (LOX)-catalyzed arachidonic and linoleic acid metabolism $(76,77)$. Based on the experiment in $C$. sinensis-infested mouse liver tissues, expressions of COX-2 and 5-LOX with increased 8-OxodG accumulation in the nucleus of the cells with inflammation were intensively detected in the inflammatory nidus (47). COX-2, an inducible form of COX, is stimulated by cytokines and lipopolysaccharide and mainly expressed during the inflammation responsible for stimulating cell growth (78). In case of RNS, N-nitrosodimethylamine (NDMA), one of the products of endogenous nitrosation, is significantly metabolized by cytochrome P-450. At intracellular level, exposure of HEK293T to ESPs of $C$. sinensis with NDMA is responsible for proliferation in the G2/M phase and expression of cell cycle related proteins, such as E2F1, phosphorylated RB and cyclin B $(42,79)$. In a study on Syrian golden hamster (experimental model), the mechanical and chemical irritation caused by $C$. sinensis worm and NDMA was considered as a probable cause for genetic alterations leading to neoplastic transformation by producing aberrant proteins including a novel oncogene PSMD10, cyclin-dependent kinase 4 gene CDK4, tumor suppressor gene $p 53$ and protein retinoblastoma (RB) and leading to enhanced survival of the transformed bile duct cells through $B A X$ and caspase 9 (80). The researchers provided the evidence on coordination between changes in the levels of gene and protein expression profile and histopathological changes in C. sinensis and NDMA-induced CCA model.

\section{CONCLUDING REMARKS}

It is undeniable that DNA damage caused by C. sinensis infestation is provoked in biliary epithelia, while proper homeostatic mechanisms are dysregulated, resulting in genetic alterations that might be indigenous to the biliary tract, thus leading to malignant transformation. The implicated mechanisms in promotion of malignancy from a parasite infestation discussed in the present review includes mechanical and chemical irritation, chronic inflammation, genomic instability, transcriptiomic, proteomic and microRNA profile alterations by ESPs, and dysregulation of immune response. However, it seems that carcinogenesis associated with $C$. sinensis can be provoked by various mechanisms and may still be a colossal subject to be elucidated. Moreover, low incidence of CCA in some areas showing a high prevalence of $O$. viverrini and C. sinensis indicates that other factors are pivotally involved in cholangiocarcinogenesis. Animal studies demonstrate that, in the absence of other carcinogens, CCA is unlikely to develop into liver fluke infestation. Consequently, it is proposed that all the described possible mechanisms could be apprehended in a concert during the development of CCA. So these liver flukes are mainly promoters and not initiators of CCA. It is also necessary that, for the discovery of biomarkers for early diagnosis and discrimination of disease from HBV infection, which is highly prevalent in many clonorchiasisendemic areas, morbidity due to C. sinensis infestation and drivers of carcinogenesis by chronic infestation should be assessed. Furthermore, for control and elimination of clonorchiasis, rapid immunological tools based on the mathematical modeling need to be developed. In conclusion, this brief review provides tiny aspects on current knowledge on the association of $C$. sinensis infestations with CCA formation, and further elucidation in future experimental and clinical based researches is necessitated.

\section{ACKNOWLEDGEMENTS}

This work was partially supported by the fund from National Research Foundation of Korea (NRF) grant funded by the Korea Government (MEST) (NO. 2012R1A2A2A01014237) (JHP).

\section{REFERENCES}

1. Gibson JB and Sobin LH (1978) Histological typing of tumors of the liver biliary tract and pancreas. Geneva, World Health Organization

2. Blechacz B and Gores GJ (2008) Cholangiocarcinoma: Advances on pathogenesis, diagnosis, and treatment. Hepatol 48, 308-321

3. Bouvard V, Baan R, Straif K et al (2009) A review of human carcinogens - Part B: Biological agents. Lancet Oncol 10, 321-322

4. Sripa B, Kaewkes S, Sithithaworn P et al (2007) Liver fluke 
induces cholangiocarcinoma. PLoS Med 4, e201

5. Oh J-K and Weiderpass E (2014) Infection and cancer: Global distribution and burden of diseases. Ann Glob Health 80, 384-392

6. Choi D, Lim JH, Lee KT et al (2006) Cholangiocarcinoma and Clonorchis sinensis infection: A care-control study in Korea. J Hepatol 44, 1066-1073

7. Lim MK, Ju YH, Franceschi S et al (2006) Clonorchis sinensis infection and increasing risk of cholangiocarcinoma in the Republic of Korea. Am J Trop Med Hyg 75, 93-96

8. Honjo S, Srivatanakul P, Sriplung H et al (2005) Genetic and environmental determinants of risk for cholangiocarcinoma via Opisthorchis viverrini in a densely infested area in Nakhon Phanom, northeast Thailand. Int J Cancer $117,854-860$

9. International Agency for Research on Cancer (2011) Monographs on the evaluation of carcinogenic risks to humans. A review of carcinogen - Part B: Biological Agents. Lyon, France

10. Shin H-R, Oh J-K, Lim MK et al (2010) Descriptive epidemiology of cholangiocarcinoma and clonorchiasis in Korea. J Korean Med Sci 25, 1011-1016

11. Viranuvatti V, Kshemsant D and Bhamarapravati N (1955) Retention cyst of liver caused by opisthorchiasis associated with carcinoma; Case report. Am J Gastroenterol 23, 442-446

12. Hou PC (1956) Relationship between primary carcinoma of the liver and infestation with Clonorchis sinensis. J Pathol Bacteriol 72, 239-246

13. Huang S-Y, Zhao G-H, Fu B-Q et al (2012) Genomics and molecular genetics of Clonorchis sinensis: Current status and perspectives. Parasitol Int 61, 71-76

14. Vennervald BJ and Polman K (2009) Helminths and malignancy. Parasite Immunol 31, 686-696

15. Lim MK, Ju Y-H, Franceschi S et al (2006) Clonorchis sinensis infection and increasing risk of cholangiocarcinoma in the Republic of Korea. Am J Trop Med Hyg 75, 93-96

16. Sripa B, Brindley PJ, Mulvenna J et al (2012) The tumorigenic liver fluke Opisthorchis viverrini - Multiple pathways to cancer. Trends Parasitol 28, 395-407

17. Chai JY, Murrell KD and Lymbery AJ (2005) Fish-borne parasitic zoonoses: Status and issues. Inter J Parasitol 35, 1233-1254

18. Hong S-T and Fang Y (2012) Clonorchis sinensis and clonorchiasis, an update. Parasitol Int 61, 17-24

19. Qian M-B, Utzinger J, Keiser J and Zhou X-N (2016) Clonorchiasis. Lancet 387, 800-810

20. Kim JH, Choi MH, Bae YM et al (2011) Correlation between discharged worms and fecal egg counts in human clonorchiasis. PLoS Negl Trop Dis 5, e1339

21. Qiao T, Ma RH, Luo XB et al (2012) Cholecystollithiasis is associated with Clonorchis sinensis infection. PLoS One 7, e42471

22. Wills-Karp M, Santeliz J and Karp CL (2001) The germless theory of allergic disease: Revisiting the hygiene hypothesis. Nat Rev Immunol 1, 69-75

23. Okada H, Kuhn C, Feillet H and Bach J-F (2010) The 'hygiene hypothesis' for autoimmune and allergic diseases: An update. Clin Exper Immunol 160, 1-9

24. Fallon PG and Mangan NE (2007) Suppression of $T_{H} 2$-type allergic reactions by helminth infection. Nat Rev Immunol $7,220-230$

25. Jeong Y-I, Kim SH, Ju JW et al (2011) Clonorchis sinensisderived total protein attenuates airway inflammation in murine asthma model by inducing regulatory $\mathrm{T}$ cells and modulating dendritic cell functions. Biochem Biophys Res Comm 407, 793-800

26. Jeong Y-I, Kim YJ, Ju JW et al (2014) Identification of anti-allergic effect of Clonorchis-derived protein venom allergen-like proteins (CsVAL). Biochem Biophys Res Comm 445, 549-555

27. Dunne DW and Cooke A (2005) A worm's eye view of the immune system: Consequences for evolution of human autoimmune diseases. Nat Rev Immunol 5, 420-426

28. Rim HJ, Lyu KS, Lee JS et al (1981) Clinical evaluation of the therapeutic efficacy of paraziquantel (Embay 8440) against Clonorchis sinensis infection in man. Ann Trop Med Parasitol 75, 27-33

29. Ambroise-Thomas P and Goullier A (1984) Parasitological examinations and immunodiagnostic advances in fluke infection. Arzneimittelforschung 34, 1129-1132

30. Kang J-M, Ju H-L, Lee J et al (2015) Mapping the putative epitope domain of Clonorchis sinensis paramyosin (CsPmy) recognized by CsPmy-specific immunoglobulin $\mathrm{G}$ in sera of human clonorchiasis. Mol Biochem Parasitol 201, 66-71

31. Kim TI, Na BK and Hong SJ (2009) Functional genes and proteins of Clonorchis sinensis. Korean J Parasitol 47 (Suppl), S59-68

32. Park GM (2007) Genetic comparison of liver flukes, Clonorchis sinensis and Opisthorchis viverrini based on rDNA and mtDNA gene sequences. Parasitol Res 100, 351-357

33. Lim JH (1990) Radiologic findings of clonorchiasis. Am J Roentgenol 155, 1001-1008

34. Xiao SH, Hui-Ming W, Tanner M et al (2005). Tribendimidine: A promising, safe and broad-spectrum anthelmintic agent from China. Acta Trop 94, 1-14

35. Echaubard P, Sripa B, Mallory FF and Wilcox BA (2016) The role of evolutionary biology in research and control of liver flukes in southeast Asia. Infect Genet Evol [Epub ahead of print]

36. Korean National Research Institute of Health, Centers for Disease Control \& Prevention (2013) Prevalence of intestinal parasitic infections in Korea - The $8^{\text {th }}$ report

37. Ministry of Health and Welfare, Korea Association of Health Promotion (2004) Prevalence of intestinal parasitic infection in Korea - The $7^{\text {th }}$ report

38. Jeong Y-I, Shin H-E, Lee S-E et al (2016) Prevalence of Clonorchis sinensis infection among residents along 5 major rivers in the Republic of Korea. Korean J Parasitol $54,215-221$

39. Li T, He S, Zhao G et al (2010) Major trends in human parasitic diseases in China. Trends Parasitol 26, 264-270

40. Hong ST, Huh S, Kho WG et al (1990) Changes of histopathological and serological findings of the liver after treatment in rabbit clonorchiasis. Seoul J Med 31, $117-127$

41. Min HK (1984) Clonorchis sinensis: Pathogenesis and 
clinical features of infection. Arzneimittelforschung 34, 1151-1153

42. Kim YJ, Choi MH, Hong ST et al (2008) Proliferative effects of excretory/secretory products from Clonorchis sinensis on the human epithelial cell line HEK293 via regulation of the transcription factor E2F1. Parasitol Res $102,411-417$

43. Kim YJ, Choi MH, Hong ST et al (2009) Resistance of cholangiocarcinoma cells to parthenolide-induced apoptosis by the excretory-secretory products of Clonorchis sinensis. Parasitol Res 104, 1011-1016

44. Yoo WG, Kim D-W, Ju J-W et al (2011) Developmental transcriptomic features of the carcinogenic liver fluke, Clonorchis sinensis. PLoS Neg Trop Dis 5, e1208

45. Fava G and Lorenzini I (2012) Molecular pathogenesis of cholangiocarcinoma. Int J Hepatol Article 2012, 630543

46. Kawanishi S and Hiraku Y (2006) Oxidative and nitrative DNA damage as biomarker for carcinogenesis with special reference to inflammation. Antioxid Redox Signal 8, 1047-1058

47. Maeng A, Lee HW, Bashir Q et al (2016) Oxidative stress-mediated mouse liver lesions caused by Clonorchis sinensis infection. Int J Parasitol 46, 195-204

48. Kawai T and Akira S (2011) Toll-like receptors and their crosstalk with other innate receptors in infection and immunity. Immunity 34, 637-650

49. Yan C, Li X-Y, Li B et al (2015) Expression of Toll-like receptor (TLR) 2 and TLR4 in the livers of mice infected by Clonorchis sinensis. J Infect Dev Ctries 9, 1147-1155

50. Liu Q and Ding JL (2016) The molecular mechanisms of TLR-signaling cooperation in cytokine regulation. Immunol Cell Biol [Epub ahead of print]

51. Choi YK, Yoon Bl, Won YS et al (2003) Cytokine responses in mice infected with Clonorchis sinensis. Parasitol Res 91, 87-93

52. Kim EM, Bae YM, Choi MH and Hong ST (2012) Cyst formation, increased anti-inflammatory cytokines and expression of chemokines support for Clonorchis sinensis infection in FVB mice. Parasitol Int 61, 124-129

53. Bhamarapravati $N$, Thammavit $W$ and Vajrasthira S (1978) Liver changes in hamsters infected with a liver fluke of man, Opisthorchis viverrini. Am J Trop Med Hyg 38, 356-362

54. Choi BI, Han JK, Hong ST and Lee KH (2004) Clonorchiasis and cholangiocarcinoma: Etiologic relationship and imaging diagnosis. Clin Microbiol Rev 17, 540-552

55. Choi M-H, Park IC, Li S and Hong S-T (2003) Excretorysecretory antigen is better than crude antigen for the serodiagnosis of clonorchiasis by ELISA. Korean J Parasitol 41, 35-38

56. Pak JH, Kim D-W, Moon JH et al (2009) Differential gene expression profiling in human cholangiocarcinoma cells treated with Clonorchis sinensis excretory-secretory products. Parasitol Res 104, 1011-1016

57. Kim D-W, Kim J-Y, Moon JH et al (2010) Transcriptional induction of minichromosome maintenance protein 7 (Mcm7) in human cholangiocarcinoma cells treated with Clonorchis sinensis excretory-secretory products. Mol Biochem Parasitol 173, 10-16

58. Pak JH, Moon JH, Hwang SJ et al (2009) Proteomic analysis of differentially expressed proteins in human cholangiocarcinoma cells treated with Clonorchis sinensis excretory-secretory products. J Cell Biochem 108, 1376-1388

59. Pak JH, Kim IK, Kim SM et al (2014) Induction of cancer-related microRNA expression profiling using excretory-secretory products of Clonorchis sinensis. Parasitol Res 113, 4447-4455

60. Ju JW, Joo HN, Lee MR et al (2009) Identification of a serodiagnostic antigen, legumain, by immunoproteomic analysis of excretory-secretory products of Clonorchis sinensis adult worms. Proteomics 9, 3066-3078

61. Khurana S, Dubey ML and Malla N (2005) Association of parasitic infections and cancers. Indian J Med Microbiol 23, 74-79

62. Oshima H, Bandaletova TY, Brouet I et al (1994) Increased nitrosamine and nitrate biosynthesis mediated by nitric oxide synthase induced in hamsters infected with liver fluke (Opisthorchis viverrini). Carcinogenesis 15, 271-275

63. Yang Q-L, Shen J-Q, Xue Y et al (2015) Pathological lesions and inducible nitric oxide synthase expressions in the liver of mice experimentally infected with Clonorchis sinensis. Korean J Parasitol 53, 777-783

64. Pinlaor S, Ma N, Hiraku Y et al (2004) Repeated infection with Opisthorchis viverrini induces accumulation of 8-nitroguanine and 8-oxo-7,8-dihydro-2'-deoxyguanine in the bile duct of hamsters via inducible nitric oxide synthase. Carcinogenesis 25, 1535-1542

65. Nam J-H, Moon JH, Kim IK et al (2012) Free radicals enzymatically triggered by Clonorchis sinensis excretorysecretory products cause NF- $\kappa \mathrm{B}$-mediated inflammation in human cholangiocarcinoma cells. Int J Parasitol 42, 103-113

66. Esquela-Kerscher A and Slack FJ (2006) Oncomirs MicroRNAs with a role in cancer. Nat Rev 6, 259-269

67. Namwat N, Chusorn P, Loilome W et al (2012) Expression profiles of oncomir miR-21 and tumor suppressor let-7a in the progression of opisthorchiasis-associated cholangiocarcinoma. Asian Pac J Cancer Prev 13, 65-69

68. Braconi C, Huang $N$ and Patel T (2010) MicroRNAdependent regulation of DNA methyltransferase- 1 and tumor suppressor gene expression by interleukin- 6 in human malignant cholangiocyte. Hepathol 51, 881-890

69. Oshima $\mathrm{H}$ and Bartsch $\mathrm{H}$ (1994) Chronic infectious and inflammation processes as cancer risk factors: Possible role of nitric oxide in carcinogenesis. Mutat Res 305, 253-264

70. Weitzman SA and Gordon LI (1990) Inflammation and cancer: Role of phagocyte-generated oxidants in carcinogenesis. Blood 76, 655-663

71. Apte RN and Voronov E (2002) Interleukin-1 - A major pleiotropic cytokine in tumor host interactions. Cancer Biol 12, 277-290

72. Ishimura N, Bronk SF and Gores GJ (2004) Inducible nitric oxide synthase upregulates cyclooxygenase-2 in mouse cholangiocytes promoting cell growth. Gastrointest Liver Physiol 287, G88-G95

73. Lowenstein CJ and Padalko E (2004) iNOS (NOS2) at a glance. J Cell Sci 117, 2865-2867

74. Bartsch $\mathrm{H}$ and Nair J (2006) Chronic inflammation and 
oxidative stress in the genesis and perpetuation of cancer: Role of lipid peroxidation, DNA damage and Repair. Langenbeck's Arch Surg 391, 499-510

75. Reuter S, Gupta SC, Chaturveri MM and Aggarwal BB (2010) Oxidative stress, inflammation and cancer: How are they linked? Free Radical Biol Med 49, 1603-1616

76. Fitzpatrick FA (2001) Inflammation, carcinogenesis and cancer. Int Immunopharmacol 1, 1651-1667

77. Furstenberger G, Krieg $P$, Muller-Decker $K$ and Habenicht AJR (2006) What are cyclooxygenases and lipoxygenases doing in the driver's seat of carcinogenesis? Int J Cancer $119,2247-2254$

78. Brown JR and DuBois RN (2005) COX-2: A molecular target for colorectal cancer prevention. J Clin Oncol 23, 2840-2855

79. Kim EM, Kim JS, Choi MH et al (2008) Effects of excretory/secretory products from Clonorchis sinensis and the carcinogen dimethylnitrosamine on the proliferation and cell cycle modulation of human epithelial HEK293T cells. Korean J Parasitol 46, 127-132

80. Uddin $\mathrm{MH}$, Choi $\mathrm{MH}$, Kim WH et al (2015) Involvement of PSMD10, CDK4 and tumor suppressors in development of intrahepatic cholangiocarcinoma of Syrian golden hamsters induced by Clonorchis sinensis and $\mathrm{N}$ nitrosodimethylamine. PLoS Negl Trop Dis 9, e0004008 